\title{
Digital Character Design of Diacritical DCROAT MARK
}

\author{
TURCIC, M.; KOREN, T. \& RUDOLF, M.
}

Abstract: This article aims at contributing to the quantitative and qualitative discussion on the design of modern fonts by analyzing the Croatian and Vietnamese dcroat sign representing a unique case of typographic solution. The main issue is the problem of designing letters that are composed of a body and a diacritical mark and that are not present in other Latin scripts. The goal of a letter design is recognition, and diacritical marks must assume eigenvalue of the letterform from the font-family concerned. The following measurements of the dcroat character were conducted: thickness, vertical and horizontal positioning. In order to enable proper diacritical design and achieve legibility, most common practices were established by measuring geometry in different fonts and font-families.

Key words: diacritics, dcroat, character, legibility, geometry
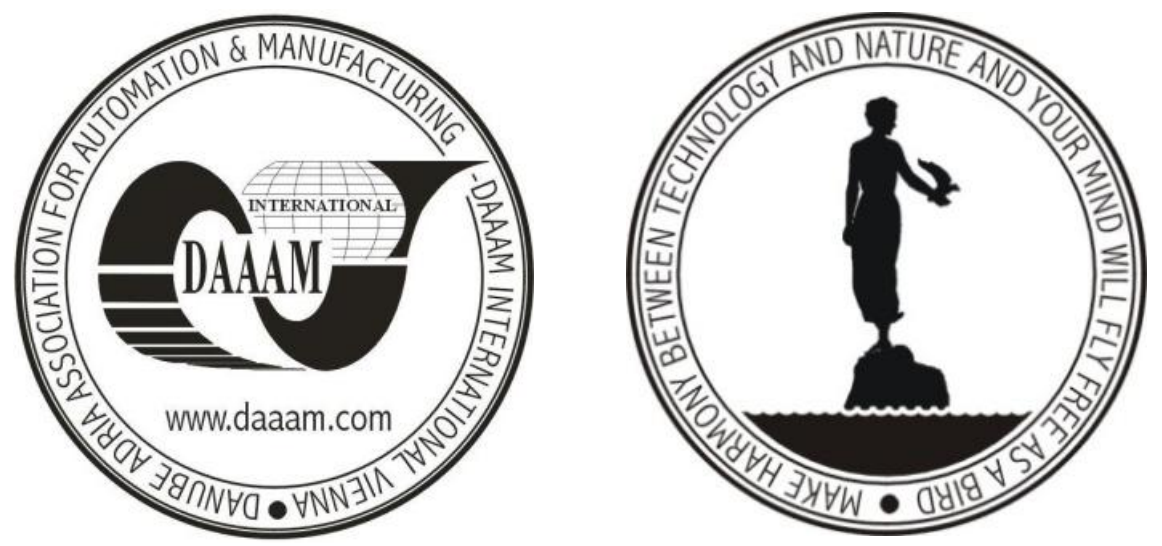

Authors' data: Dipl.-Ing. Turcic, M[aja]*; Dipl.-Ing. Ph.D. Koren, T[ajana]**; Dipl.-Ing. Rudolf, M[aja]**, *Polytechnic of Zagreb, Vrbik 8, 10000, Zagreb, Croatia, **University of Zagreb, Faculty of Graphic Arts, Getaldiceva 2, 10000, Zagreb,Croatia,mturcic@tvz.hr, tkoren@grf.hr, mrudolf@grf.hr

This Publication has to be referred as: Turcic, M[aja]; Koren, T[ajana] \& Rudolf, M[aja] (2011). Digital Character Design of Diacritical Dcroat Mark, Chapter 32 in DAAAM International Scientific Book 2011, pp. 409-416, B. Katalinic (Ed.), Published by DAAAM International, ISBN 978-3-901509-84-1, ISSN 1726-9687, Vienna, Austria

DOI: 10.2507/daaam.scibook.2010.32 
Turcic, M.; Koren, T. \& Rudolf, M.: Digital Character Design of Diacritical Dcroat...

\section{Introduction}

The number of digital fonts has been increasing constantly. Owing to the available digital technology, 'old fonts' are being digitalized by the type foundries, while new fonts are also being created. Problems that type designers have to deal with derive from a huge number of glyphs which are considered a standard part of fonts. Besides characters and symbols, they include characters with diacritical marks from different scripts (i.e. Asia, South America, Greece, Eastern Europe, etc.)

When the Unicode Standard was introduced, it enabled the use of any glyph for any linguistic particularity. Most special characters have a long cultural history generally unknown to type designers. That is why it is important to educate type designers and typographers and raise their consciousness enabling them to produce or repair special characters correctly. That is hard to accomplish for an obscure letter like the lowercase dcroat present only in Croatian and Vietnamese.

When designing diacritics, it is necessary to pay attention to their thickness, position, size and eigenvalue of the letterform from the font-face concerned. It is also relevant to plan ahead for all the font-faces and possible spacing issues. In order to enable proper diacritical design and achieve legibility, the most common practices and mistakes were established by measuring geometry of the dcroat diacritical mark in different font faces.

\section{Diacritical mark design}

In editing and graphic design, a good typographic design can be achieved only if both texts and fonts are regularly edited. Fonts are mostly not ready for the general use when they first reach the market, so they should be carefully edited for the purpose of a certain text/script. The way diacritics are made depends on the linguistic diversity of designers. If a special character is missing from the font, it shouldn't be difficult to produce one from the beginning. (Bringhurst, 2000)

In spite of a large number of fonts available today, there are still many that do not support European languages, let alone African or Asian. In Latin, diacritics are used to add new compound characters to represent sounds. Some of the biggest font foundries still use the basic set of accents when designing fonts. This often results in an inadequate design and confusion of type designers. Letters with diacritics must offer readability and aesthetic value to the native reader. The lack of stylistic harmony is most visible in serif fonts. The basic letter of serif fonts has a certain style and contrast that is missing in the diacritic design. The accents should not differ from the design of the given typeface, either by size or thickness. Special attention should be given to small sizes of letters (8-12pt) because of the potential overlapping and the lack of recognition. Positioning depends on the letter size (display/text) and the fontfamily type (regular/bold). Thickness should be also considered. Diacritics are smaller in size than letters, so their contrast should not be lighter or darker but proportionally smaller. Kerning should be done carefully to avoid overlapping or setting letters too far apart. (Březina, 2009)

Owing to the available digital technology, it is possible to create and use all needed scripts. Complete fonts including all characters for European and most scripts 
from around the world can be bought. However, illiteracy can still be noticed among typographers, journalist and designers, because of the 'fear' of characters with diacritics. Commercial fonts contain many characters, but often lack small caps, old style figures, ligatures and characters with diacritics.

Elements needed for creating rare characters, especially those from Eastern Europe, can usually be found within the font concerned. An educated typographer, using adequate software, should be able to create those characters. However, if these characters are present, but misshaped in any way, they should be corrected with confidence and skill. (Bringhurst, 2005)

With the increasing number of available fonts, mostly 'English' oriented, inadequate and incomplete fonts are the starting point for typographers in need of rare linguistic characters. Enough information on rare special characters should be provided in order to accomplish linguistic accuracy and create more complete fonts. Each alphabet depends on the respective culture evolving for hundreds of years. Type designers and typographers should thoroughly analyze all linguistic specifications and technical appearance of rare characters needed by the non-English speaking domain. Typical Latin alphabet consists of around 600 characters, but with all the glyphs borrowed from other scripts (i.e. Greek, technical symbols, etc.) it exceeds the 1000 characters. There will always be the need to create or edit rare characters from the existing fonts. Diacritics are mostly misshaped, inadequate in size or position. (Bringhurst, 2000)

Microsoft's Character Design Standards is the only official and available resource for the diacritic design. Its purpose is to compile general rules for character design in Latin scripts. Descriptions usually concentrate on the diacritic positioning and not the design itself. The biggest sources of information are the fonts themselves, but they have to be considered with caution because they can be misleading. Diacritical marks are signs added to a certain letter to change its meaning or pronunciation.

The most common diacritics are: acute, grave, circumflex, dieresis and tilde (present in all major cultures: French, Spanish and German). In the Croatian language there are three types of diacritics: acute (present on letter c), caron (present on letters $\mathrm{c}, \mathrm{s}$ and $\mathrm{z}$ ) and bar (present on letter d). Since the diacritic caron is actually the inverted version of circumflex and both acute and circumflex can be found in most Western European fonts the basic knowledge in type production should be sufficient for creating all Croatian letters with diacritics, except the dcroat letter.

The bar in the dcroat letter can be categorized as: through (positioned through the letter, attached to the letter); asymmetrical and non-centered (base dependant). Diacritic design problems in the dcroat letter are: asymmetry, width harmony and cultural preferences.

In asymmetrical letters, the balance issue refers to finding the optical center of the letter. If the diacritical mark is misbalanced, the letter will seem to gravitate to a certain side. Letters with counters usually have their optical balance around the center of the counter. When the diacritic is properly positioned, the letter must not collide with the next letter. The width, advance and kerning of the letter must be adjusted to avoid collision. In bold faces and sans-serif fonts, there is even less space than usually given by the serif. The most common solution for the dcroat letter is to 
Turcic, M.; Koren, T. \& Rudolf, M.: Digital Character Design of Diacritical Dcroat...

shorten the diacritic itself. Size and weight of diacritics are crucial for recognition, without which the context and meaning of words can alter. On the other hand, if the bar is too thick it can merge with the serif or the bowl. The biggest problem is the lack of standards. Western oriented type designers cannot be expected to be informed of Eastern European cultural and linguistic specifications. With digital capabilities, solving this problem is possible but rarely accurate. The necessary knowledge and information on diacritics design should be available.

As the current trend of the increasing multilingual demands for fonts continue, type designers are challenged to evolve, not just in the design area, but even more in the technical segment. The expansion of required fonts that support Eastern European sets of characters results in those sets of character becoming standard, rather than extended versions. Along with the knowledge of designing standard letters, a designer should understand the terms such as Unicode or OpenType. Those technologies are needed to properly support scripts rich with diacritics, and therefore designers should be educated to use them.

People worldwide should be given the possibility to use all special characters included in their native language. Today, there are a few designers who have the extensive knowledge and will to create complete and appropriate fonts. Extensive research and expert publications are required to fulfill those needs. Owing to rapid technological growth and availability, the standards for specific type design remain feeble. (J. Victor Gaultney, 2002)

\section{History and design}

The introduction of the letter $\mathrm{d}$ with a stroke in Croatian alphabet was proposed by Đuro Daničić in the late $19^{\text {th }}$ century. The idea was to represent one phoneme with one glyph.

In the time of the lead type, the creation and design of letters was solely dependent on type foundries. These foundries, such as Monotype and Linotype, had all the copyrights of these fonts. Given that, at that time, there were no type foundries in Croatia, Croatian printing houses ordered their sets of fonts from the Czech Republic or Germany. This means that the design of Croatian letters with diacritics was not dependant on Croatian typographers, designers or printing houses, but foreign type foundries where those fonts were made. In the time of phototypesetting, the computerization process was taken over by the big type foundries with the respective copyrights. However, with the need of digitalizing fonts to PostScript format, professors of the Faculty of Graphic Arts, Vilko Žiljak and Klaudio Pap, undertook that task. In 1990, they digitalized over 3000 fonts that worked on all the major popular systems at the time and determined the coding places for all the Croatian letters. These fonts were used in newspaper publishing all over Yugoslavia. (Žiljak,V., Pap,K., Žiljak,D., 1990) These Croatian letters were the first attempt to design diacritics by Croatian authors and remained in use until large corporations, such as Microsoft and Adobe, concerted to the Unicode standard, beginning to include letters with diacritics in some of the fonts that they produced.

According to Microsoft (http://www.microsoft.com/typography/developers /fdsspec/lowercase.htm) the lowercase letter d with a stroke (also known as dyet or 
dcroat) is used in Croatian, Vietnamese, Macedonian and Serbian language. Its design is based on the lowercase $d$ with an added bar. The bar should be of the same thickness as other lowercase characters with bars. Vertically the bar should be visually centered between the $\mathrm{x}$-height and the ascender. It should horizontally extend to the right of the stem similarly to the serif in serif designs and enough to be visible at small size but not too long to cause spacing problems for sans serif designs. To the left, the bar should extend approximately one half the width of the lower bowl of the $\mathrm{d}$ and in italic designs visually one half the bowl. The advance width should be the same as the lowercase d. In sans serif designs, it may be necessary for the advance width of this character to be greater than the lowercase $d$ to visually compensate for the bar. Croatian typographer Nikola Đurek adds that the thickness and the position of the bar should be determined by the contrast of the letter and the $\mathrm{x}$-height. The bar shouldn't be too short, the ideal length starts with the optical center of the letter. (Đurek, 2009) The capital letter dcroat (Đ) looks the same as the Icelandic Eth, so it will not be discussed in this article.

When the 7-bit ASCII code was used, it was not possible to use the dcroat letter due to the lack of space. There was enough space for 94 visible characters that included 26 upper-case and 26 lower-case characters, numbers, basic punctuation and some other marks. When the extended 8-bit system was introduced, another 128 spaces became available. The position of the dcroat letter in all the major code systems (MS Windows 1250, IBM-1129, ISO-8859 Latin 2, Mac OS Croatian) was 240 (decimal) or F0 (hexadecimal). This solution was still impractical because the user was forced to switch between different systems and languages. Another problem was the limited space given for all the characters still insufficient for all languages. All technical problems were solved with the 16-bit Unicode standard that supports thousands of glyphs. The hexadecimal code number of the lowercase dcroat is U+0111. (Wells, 2000)

\section{Measurements}

By measuring geometry of the diacritical mark of the dcroat letter from different fonts and font faces, most common practices in designing were established. Different results were expected in serif and sans-serif fonts. Three values were measured:

1. Extension to the left (50\% of counter was expected)

2. Vertical position (50\% of ascender was expected)

3. Thickness (depending on the contrast)

\begin{tabular}{|l|l|l|l|l|}
\hline Font & $\mathrm{x} \%$ & $\mathrm{~h} \%$ & $\mathrm{H} \%$ & $\mathrm{~d} \%$ \\
\hline Serif-regular & 61 & 50 & 38 & 136 \\
\hline Sans-serif-regular & 55 & & 44 & 119 \\
\hline Serif-bold & 78 & 51 & 37 & 123 \\
\hline Sans-serif bold & 67 & & 45 & 117 \\
\hline
\end{tabular}

Tab. 1 Results 


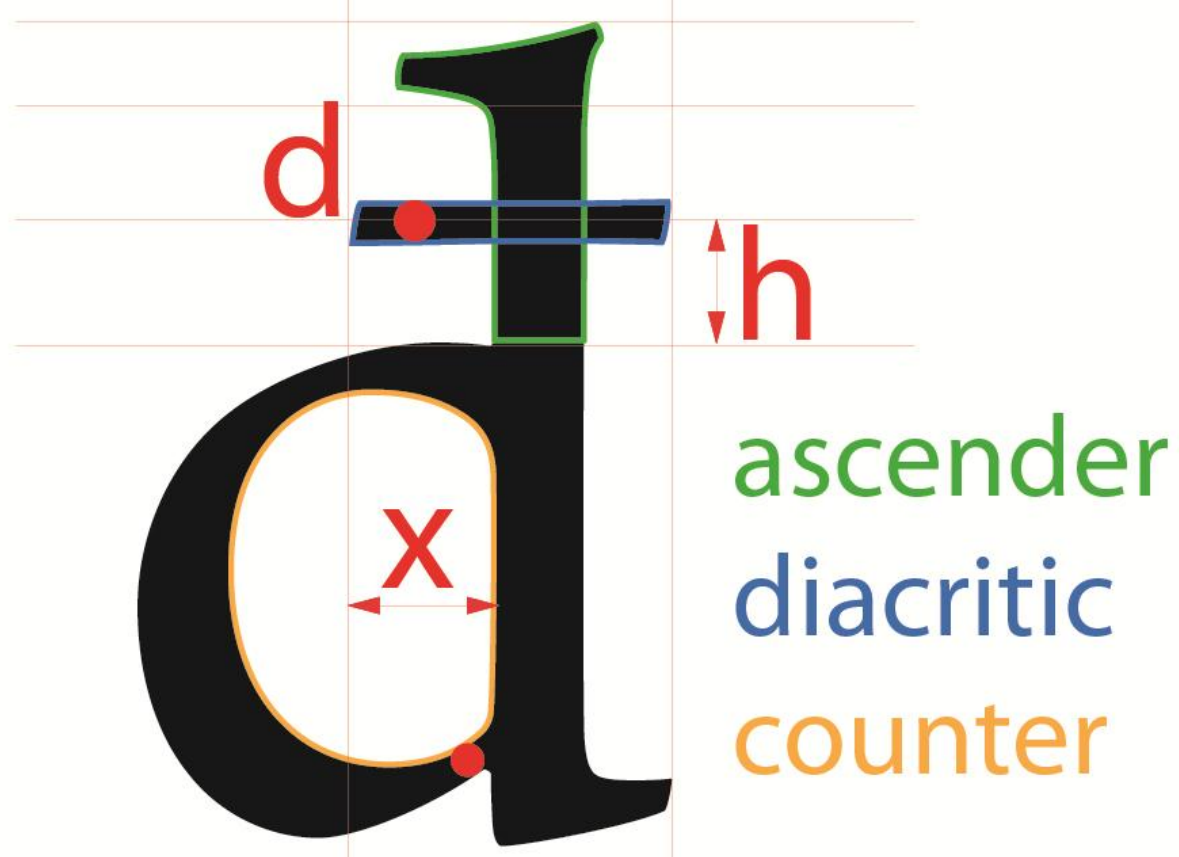

Fig.1 dcroat construction

The measurements prove that the bar extends to the left more than $50 \%$ of the counter, meaning that greater importance is given to the optical center of the letter than the mathematical one. Because of greater contrasts, serif fonts have different optical centers, found further to the left. The results found in sans-serif fonts were closer to the expected values, mostly because of smaller contrasts. Therefore, the following designs are not adequate (the bar is too long or too short): Excelsior (77\%), Garamond (49\%), Lucida Sans (71\%) and Neutra (41\%).

The vertical position of the bar in serif fonts is mostly around $50 \%$ of the ascender, excluding the height of serifs. The bar is expected to be placed in the middle between the bowl and the serif. In sans-serif fonts the bar is placed in the middle between the bowl and the overall height of the ascender (for the lack of serifs). The average is less than 50\%, depending on the $\mathrm{x}$-height and the optical alignment. Vertical misplacements can be found in Garamond (56\%), Corbel (52\%) and Haarlamer sans $(28 \%)$.

The thickness of the bar is thicker than the thinnest part of the letter, and in serif fonts it is even more visible due to bigger contrasts. When determining the thickness of the bar, the contrast and the overall design of the base letter should be considered. Mistakes are more visible in bold faces, especially in serif fonts.

Different rules apply in the measurements of bold faces. The extension to the left is much more than 50\%, around $67 \%$ in sans-serif fonts, and $78 \%$ in serif ones, mostly because of the bigger contrasts of letters. The numbers are statistically obtained, therefore not realistic. It cannot be expected that the bar extends to the half of the counter because the bowl is wider and depends on the design of the letter $d$ in the given font-face. Inappropriate lengths of the bar can be seen in Trebuchet and Neutra fonts, where the bar is too short and can cause recognition problems in small sizes. 

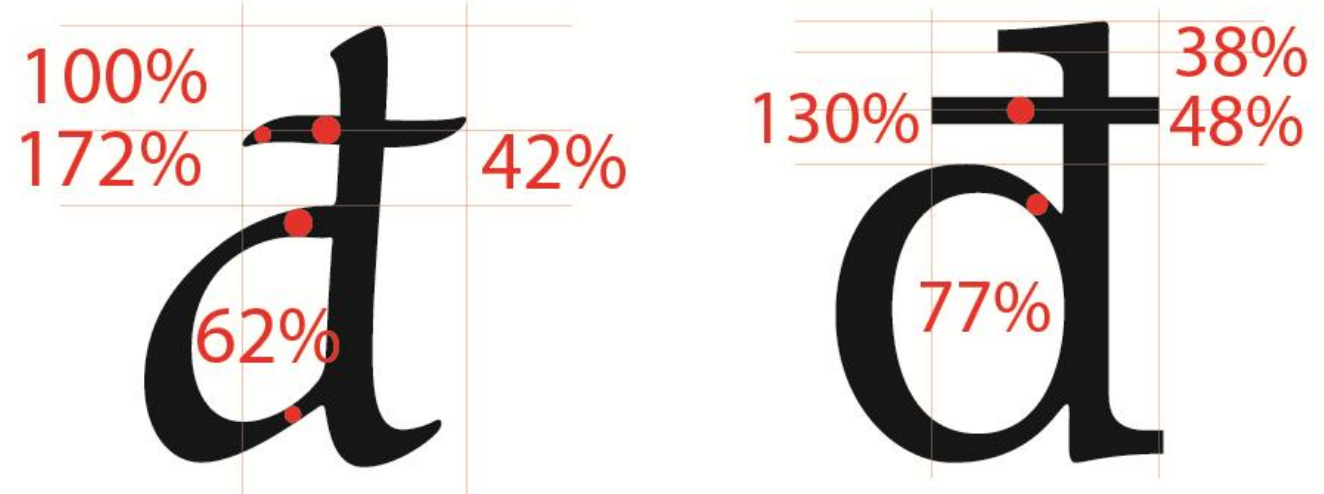

sanvito

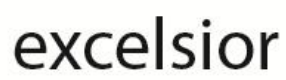

Fig. 2 Appropriate vs. inappropriate examples through measurements

arno

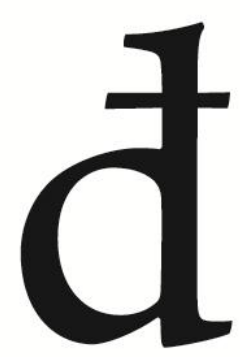

excelsior

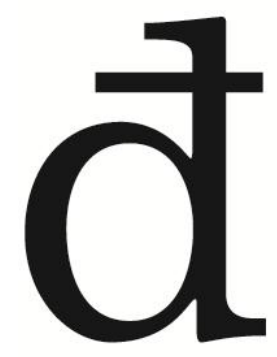

candara

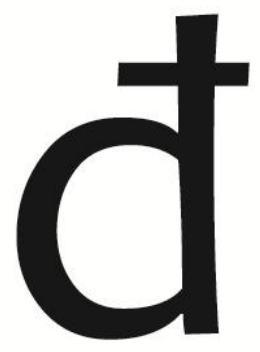

garamond

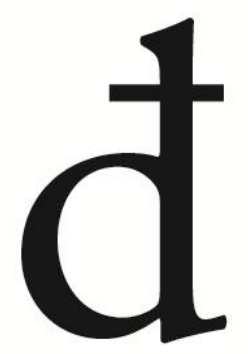

warnock

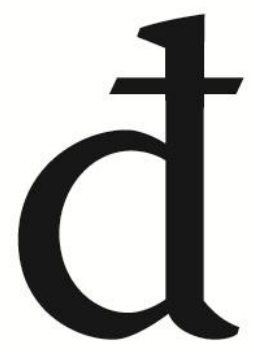

lucida sans

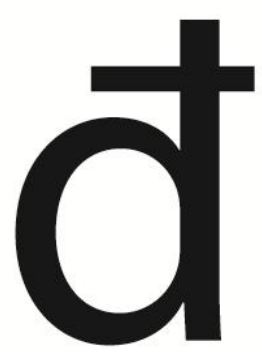

sanvito

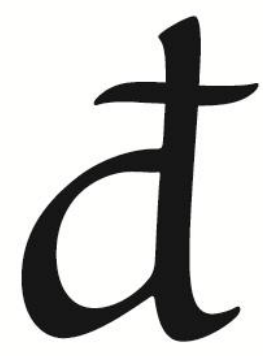

neutra

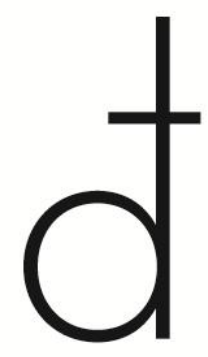

Fig. 3 Top: good solutions; bottom: poor solutions

Vertical positioning in bold faces remains in the optical middle of the ascender. Problems regarding the thickness of the bar, the serif and the x-height demand for different solutions because of the overlapping possibilities in smaller sizes. An alternative solution was proposed by the designer of Gill Sans Ultra Bold font where the bar is positioned on top of the ascender. Inadequate thickness can be found in Chaparral bold and Verdana bold fonts. Optimal solutions, in length and thickness, are offered in Constantia, Century and Candara. 
Turcic, M.; Koren, T. \& Rudolf, M.: Digital Character Design of Diacritical Dcroat...

\section{Conclusion}

The goal of the font design is recognition and legibility. However, there is insufficient information on the appearance of rare characters not present in the Western cultures, especially Eastern European letters composed of the body and diacritical marks. Designing the dcroat letter consisting of the small letter $d$ with an added bar present only in Croatian and Vietnamese scripts is an example of this problem. Since there is a limited amount of available relevant literature concerning Eastern European diacritical marks, this article aims at clarifying the issues of the dcroat letter design. By measuring the geometry of this diacritical mark, general rules were established concerning its width, vertical position and thickness. The most common mistakes and problems in diacritics design were discussed and solutions were proposed. Besides geometry, the style and culture should be considered as well. Nowadays, many fonts are available for usage, but a few offer appropriate and legible solutions for each special character needed in each culture and script. Discussion elaborated in this article aims at encouraging and motivating new typographical solutions and repairs of other present diacritical problems. Presented information should enable typographers to design dcroat, as well as to make them aware of the problem of missing characters and the importance of diacritics.

The research described in this paper will lead to the development of standards concerning diacritical marks present in Eastern Europe. Future research should expand to other rare characters and accented letters enabling typographers and type designers to properly design and use these glyphs although they are not acquainted with less popular cultures.

\section{References}

Březina, D. (2009) On diacritics, Available from: http://ilovetypography.com /2009/01/24/on-diacritics, Accessed on: 2009-10-01

Bringhurst, R. (2000) Editing the pretext, Journal of scholarly publishing, vol.31, No.3, April 2000, pn.113-125, ISSN: 1198-9742

Bringhurst, R. (2005) The Elements of Typographic Style, Hartley \& Marks, ISBN: 088179-206-3, Vancouver, Canada

Đurek, N. (2009) Study of technology, readability and aesthetics of Croatian script, Ph.D. Dissertation, University of Zagreb, Faculty of Graphic Arts

Gaultney, J. V. (2002) Problems of diacritic design for Latin script text faces, Available from: http://www.sil.org/ gaultney/research.html, Accessed on: 2010-05-20

Wells, J.C. (2000) Orthographic Diacritics and Multilingual Computing, Language Problems \& Language Planning, vol 24, No.3, pn. 249-72, fall 2000, ISSN: 0272-2690

Žiljak, V.; Pap, K. \& Žiljak, D. (1990) GF Postscript fonts for Macintosh and PC, Faculty of Graphic Arts, University of Zagreb

***(1998)http://www.microsoft.com/typography/developers/fdsspec/lowercase.htm -

Character design standards, Accessed on:2009-10-01 